\title{
Three-year outcome analysis of alpha I-blocker naftopidil for patients with benign prostatic hyperplasia in a prospective multicenter study in Japan
}

\author{
This article was published in the following Dove Press journal: \\ Patient Preference and Adherence \\ 22 July 2016 \\ Number of times this article has been viewed
}

\section{Naoya Masumori' \\ Taiji Tsukamoto' \\ Akihiko Shibuya ${ }^{2}$ \\ Noriomi Miyao 3 \\ Yasuharu Kunishima ${ }^{4}$ \\ Akihiko Iwasawa ${ }^{5}$ \\ 'Department of Urology, Sapporo Medical University School of Medicine, Sapporo, ${ }^{2}$ Kaguraoka Urological Clinic, Asahikawa, ${ }^{3}$ Division of Urology, Muroran City General Hospital, Muroran, ${ }^{4}$ Hokkaido Social Work Association Obihiro Hospital, Obihiro, IIwasawa Clinic, Sapporo, Japan}

Purpose: Our aim was to prospectively analyze the 3-year outcomes of naftopidil treatment for patients with benign prostatic hyperplasia (BPH), including those who dropped out during follow-up and had retreatment for BPH after termination of the drug within 3 years.

Patients and methods: Naftopidil, $50 \mathrm{mg} / \mathrm{d}$ or $75 \mathrm{mg} / \mathrm{d}$, was given to 117 patients having BPH aged 50 years and older who had international prostate symptom scores (IPSS) $\geq 8$. They were prospectively followed for 3 years with periodic evaluation. If naftopidil was terminated, the reason was determined. For patients with termination, an outcome survey was done to evaluate the status of retreatment for BPH at 3 years.

Results: Twenty-five patients (21.4\%) continued the same medication for 3 years. The total IPSS, quality of life index, BPH problem index, and maximum flow rate were significantly improved during 3 years. Treatment failure defined as symptomatic progression (an increase in the IPSS of $\geq 4$ points compared to the baseline value), development of acute urinary retention, conversion to other $\alpha 1$-blockers, add-on of a $5 \alpha$-reductase inhibitor, or conversion to surgery was observed in 41 patients (35.0\%). In the univariate analysis, age, prostate volume, and serum prostate-specific antigen were predictors of treatment failure. Of the 50 patients who discontinued naftopidil during the follow-up, only 13 (26\%) patients reported that they needed retreatment with $\alpha 1$-blockers and/or surgery within 3 years.

Conclusion: Long-term efficacy of naftopidil was observed, although older age, increased prostate volume, and elevated prostate-specific antigen at baseline were highly likely to result in treatment failure. Even after termination for various reasons, only a small portion of the patients needed retreatment for BPH within 3 years.

Keywords: benign prostatic hyperplasia, alpha 1-blocker, naftopidil, long-term outcome, treatment failure, retreatment

\section{Introduction}

We previously reported the results of a 5-year prospective study of tamsulosin, which has threefold higher affinity for $\alpha 1 \mathrm{~A}$ than for the $\alpha 1 \mathrm{D}$ subtype in patients with lower urinary tract symptoms (LUTS), suggestive of benign prostatic hyperplasia (BPH). ${ }^{1}$ In that study, we found that 1) only a small portion of patients (approximately one-third) continued the same tamsulosin monotherapy for 5 years; 2) there was a long-term efficacy of tamsulosin in patients who could continue the monotherapy; and 3) a large prostate volume (PV) and a large amount of postvoid residual urine volume (PVR) at baseline were highly likely to result in the failure of the tamsulosin monotherapy.
Correspondence: Naoya Masumori Department of Urology, Sapporo Medical University School of Medicine, SI, W16, Chuo-ku, Sapporo 060-8543, Japan

Tel $+8 \mid$ II $61|2|||$

Fax $+8 \mid$ II 6122709

Email masumori@sapmed.ac.jp 
On the other hand, the long-term outcome of naftopidil, which has threefold higher affinity for $\alpha 1 \mathrm{D}$ than for the $\alpha 1 \mathrm{~A}$ subtype, has never been prospectively investigated. In addition, although the tamsulosin study demonstrated that, aside from patients with treatment failure, $>50 \%$ of patients quitted the medication for various reasons, including symptomatic improvement, ${ }^{1}$ the clinical course after the discontinuation remained unknown. Some patients might need retreatment for recurrence of LUTS or the development of complications such as acute urinary retention (AUR) induced by BPH.

Thus, we conducted a prospective multicenter study to evaluate the long-term efficacy of naftopidil as well as to predict the risk factors for treatment failure. In addition, the reasons for discontinuation of naftopidil and the need for subsequent retreatment after discontinuation were determined.

\section{Patients and methods}

Patients having BPH/LUTS aged 50 years or older who had an international prostate symptom scores (IPSS) $\geq 8$ were enrolled in this prospective multicenter study conducted between July 2006 and January 2013 (patients were recruited until September 2009) in Hokkaido, Japan. We excluded patients with prostate cancer and diseases that affect urinary conditions such as neurogenic bladder, bacterial prostatitis and urinary tract infection, AUR, and indwelling of a urethral catheter.

The IPSS, BPH problem index (BPI), and quality of life (QoL) index were evaluated at baseline. Digital rectal examination and serum prostate-specific antigen (PSA) determination were performed to screen for prostate cancer. PV $(\mathrm{mL})$ was determined by transrectal or transabdominal ultrasound using the following formula: ${ }^{2}$

$$
\begin{aligned}
0.523 & \times \text { anteroposterior diameter }(\mathrm{cm}) \\
& \times \text { transverse diameter }(\mathrm{cm}) \\
& \times \text { longitudinal diameter }(\mathrm{cm})
\end{aligned}
$$

The patients underwent uroflowmetry to evaluate the maximum flow rate $\left(Q_{\max }, \mathrm{mL} / \mathrm{s}\right)$. PVR $(\mathrm{mL})$ was measured by transabdominal ultrasound using the same formula as for PV.

Naftopidil, $50 \mathrm{mg} / \mathrm{d}$ or $75 \mathrm{mg} / \mathrm{d}$, was given to the patients. The initial dosage of naftopidil was selected by the physician based on experience, disease severity, the patient's age, comorbidity, etc. The patients were prospectively followed for 3 years with periodic evaluations as shown in Table 1. The dosage of naftopidil was adjusted when clinically necessary based on the experience of each urologist.

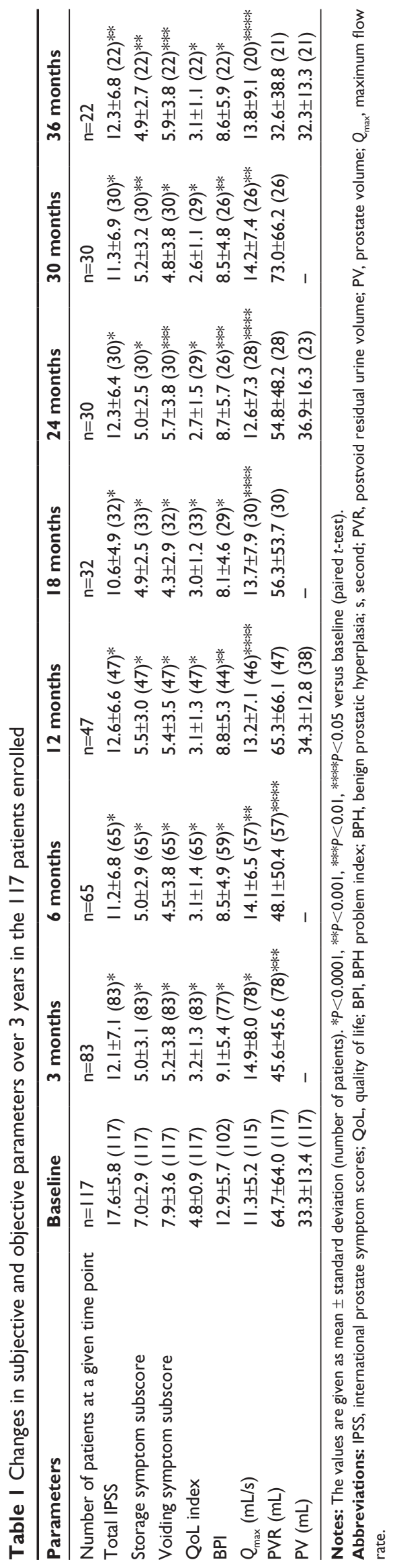


Discontinuation was defined as the sum of for-cause termination and loss to follow-up. If naftopidil had to be terminated during follow-up (for-cause termination), the reason was determined. If a patient did not come to the hospital to receive a prescription (loss to follow-up), a questionnaire asking the reason for not coming (improvement in LUTS, no change/worsening of LUTS, development of adverse events, business, others) with the IPSS, QoL index, and BPI was mailed to the patient as described in the previous report. ${ }^{1}$

Treatment failure was defined as symptomatic progression (an increase in the IPSS of $\geq 4$ points compared to the baseline value), the development of AUR, conversion to other $\alpha 1$-blockers, addition of a $5 \alpha$-reductase inhibitor (RI), or conversion to surgery. An increased dosage of naftopidil was not considered to be treatment failure in this study.

For the patients who discontinued naftopidil during follow-up, whether events such as AUR and retreatment for BPH/LUTS developed or not were evaluated at 3 years after starting the naftopidil treatment by mail or telephone survey.

Differences in parameters between two time points and two groups were determined by the paired and the unpaired $t$-tests, respectively. The Cox proportional hazards model was used to estimate the relative risks of parameters. Rate estimates were constructed using the Kaplan-Meier method. The log-rank test was used to evaluate the differences between the rates. Correlations between two parameters were determined by the Pearson product-moment correlation coefficient and evaluated by Fisher's $z$ transformation.

The present study was approved by the institutional review board of Sapporo Medical University (No 17-83). Written informed consent was obtained from all the participants.

\section{Results}

In this study, 117 patients were registered. Their mean age ( \pm standard deviation) was 68.3 years $( \pm 6.6)$. The baseline characteristics of the patients are shown in Table 1. The mean PSA was $1.8 \pm 1.5 \mathrm{ng} / \mathrm{mL}$ (data were not available for four patients). Naftopidil, $50 \mathrm{mg}$ and $75 \mathrm{mg}$, was initially given to 108 and nine patients, respectively. Among the 108 patients receiving $50 \mathrm{mg}$ of naftopidil, the dosage was increased to $75 \mathrm{mg}$ for 20 patients and decreased to $25 \mathrm{mg}$ for one patient. During the observation period, the dosage was not changed for any of the nine patients who were initially receiving $75 \mathrm{mg}$ of naftopidil.

The total IPSS, storage symptom subscore (frequency, urgency, nocturia), voiding symptom subscore (intermittency, weak stream, straining), QoL index, BPI, and $Q_{\max }$ were significantly improved during 3 years (Table 1 ). Although PVR significantly improved by 6 months, statistical significance disappeared after 1 year. There were no significant changes in PV.

Of the 117 patients who were administered naftopidil, $25(21.4 \%)$ continued the same medication for 3 years (Figure 1). The remaining 92 patients (78.6\%) discontinued the medication due to for-cause termination in 45 (38.5\%) patients and loss to follow-up in $47(40.2 \%)$ patients. The reasons for termination are indicated in Figure 1. Quitting the drug by conversion to other $\alpha 1$-blockers, addition of a $5 \alpha-\mathrm{RI}$, or conversion to surgery due to insufficient efficacy was observed in 21 (17.9\%), five (4.3\%), and seven (6.0\%) patients, respectively. The drug was terminated because of symptomatic improvement in 33 patients $(28.2 \%)$. Six patients $(5.1 \%)$ terminated medication because of adverse events (postural hypotension in two, dizziness in two, gastric discomfort in two).

Treatment failure was observed in 41 patients $(35.0 \%)$ during the 3 years of follow-up. In addition to the 33 patients mentioned earlier who had conversion to other $\alpha 1$-blockers, addition of a $5 \alpha-R I$, or conversion to surgery, eight (6.8\%) patients showed symptomatic progression defined as an increase in the IPSS of $\geq 4$ points compared to the baseline value. The baseline factors that influenced treatment failure were evaluated (Table 2). In the univariate analysis, age, PV, and PSA were factors predicting treatment failure. Since PV and PSA were correlated ( $r=0.424, P<0.0001)$, multivariate analyses were done using age and PV (multivariate 1) and age and PSA (multivariate 2). Although neither age nor PV predicted treatment failure in the multivariate 1 analysis, the baseline serum PSA value was selected as an independent factor predicting treatment failure in the multivariate 2 analysis. On the Kaplan-Meier curve, there was a significant difference in the treatment failure rates between patients aged $\geq 70$ years and those aged $<70$ years $(P=0.028$; Figure 2A) and between patients with PSA $\geq 1.8 \mathrm{ng} / \mathrm{mL}$ and those with PSA $<1.8 \mathrm{ng} / \mathrm{mL}$ ( $P=0.008$; Figure $2 \mathrm{~B})$. Although there was no significant difference, patients with $\mathrm{PV} \geq 35 \mathrm{~mL}$ tended to have treatment failure compared to those with $\mathrm{PV}<35 \mathrm{~mL}(P=0.226$; Figure $2 \mathrm{C})$.

During follow-up, outcome analysis at 3 years was performed for the 50 patients who discontinued naftopidil for various reasons (Figure 1). Of them, 37 patients (74\%) reported no events such as AUR and retreatment for BPH/ LUTS between the time of termination $(9.2 \pm 10.2$ months after administration) and 3 years in the outcome survey 


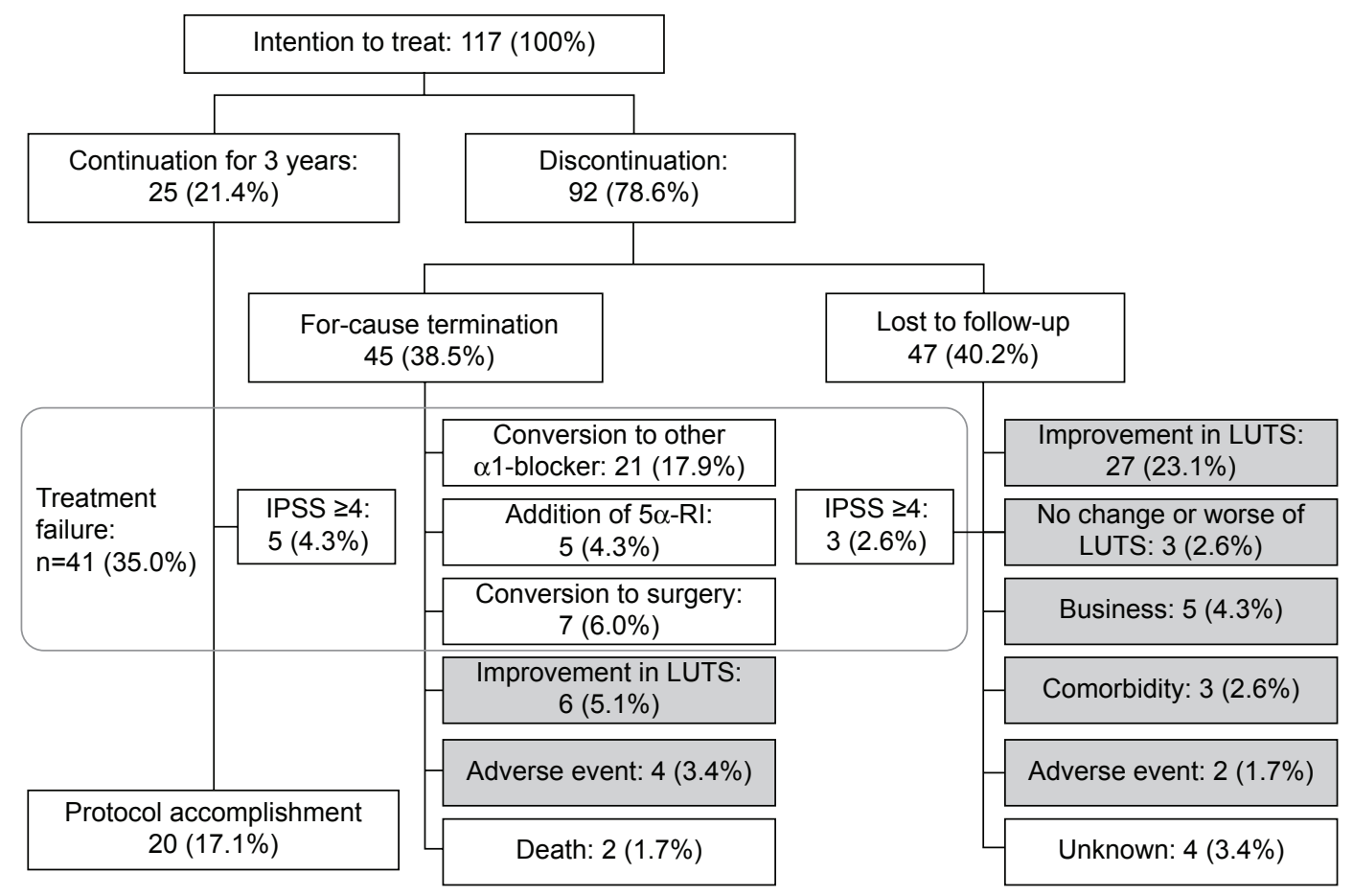

Figure I Outcomes of 117 patients until 3 years after administration of naftopidil.

Notes: Treatment failure was defined as symptomatic progression (an increase in the IPSS $\geq 4$ points compared to the baseline value), development of AUR, conversion to other $\alpha$ l-blockers, add-on of a $5 \alpha$-RI, or conversion to surgery. Gray boxes indicate 50 patients who discontinued naftopidil during follow-up and had evaluation of the outcome at 3 years after starting naftopidil treatment.

Abbreviations: IPSS, international prostate symptom scores; AUR, acute urinary retention; RI, reductase inhibitor; LUTS, lower urinary tract symptoms.

(Table 3$)$. On the other hand, 13 patients $(26 \%)$ reported that they received retreatment with $\alpha 1$-blockers and/or surgery between the time of termination $(6.7 \pm 4.8$ months after administration) and 3 years. The average time between termination and retreatment was 19.4 \pm 17.4 months.

There were 33 patients whose reason for discontinuation was improvement in LUTS (Figure 1). Compared to the remaining 84 patients, they had better $Q_{\max }$ and smaller PV at baseline (Table 4). Although statistically insignificant, the 33 patients tended to have a lower IPSS and PSA than the remaining 84 patients. Of the 33 patients, eight (24.2\%) received retreatment after discontinuation of naftopidil due to symptomatic improvement. There were no differences in the baseline age, PV, and PSA between patients with retreatment and those with no events (age, 68.5 \pm 3.7 years vs 66.5 \pm 6.9 years, $P=0.444$; PV, $29.1 \pm 8.4 \mathrm{~mL}$ vs $28.9 \pm 8.7 \mathrm{~mL}$, $P=0.964$; PSA, $1.2 \pm 1.4 \mathrm{ng} / \mathrm{mL}$ vs $1.3 \pm 1.6 \mathrm{ng} / \mathrm{mL}, P=0.822)$. Subsequent retreatment was not predicted by the baseline IPSS, QoL index, or BPI or by the IPSS, QoL index, or BPI at the last visit or after discontinuation (Table 5).

Table 2 Cox proportional hazards model to predict factors for treatment failure

\begin{tabular}{|c|c|c|c|c|c|c|c|c|c|}
\hline \multirow[t]{2}{*}{ Variables } & \multicolumn{3}{|l|}{ Univariate } & \multicolumn{3}{|l|}{ Multivariate I } & \multicolumn{3}{|l|}{ Multivariate 2} \\
\hline & Hazard ratio & $95 \% \mathrm{Cl}$ & $P$-value & Hazard ratio & $95 \% \mathrm{Cl}$ & $P$-value & Hazard ratio & $95 \% \mathrm{Cl}$ & $P$-value \\
\hline Age & 1.052 & $1.002-1.107$ & 0.042 & 1.040 & $0.987-1.096$ & 0.145 & 1.058 & $0.999-1.120$ & 0.052 \\
\hline IPSS & 0.979 & |0.929-1.03। & 0.419 & & & & & & \\
\hline BPI & 1.012 & $0.952-1.075$ & 0.710 & & & & & & \\
\hline QoL index & $\mathrm{I} .308$ & $0.899-1.902$ & 0.161 & & & & & & \\
\hline$Q_{\max }$ & 0.955 & $0.897-1.018$ & 0.160 & & & & & & \\
\hline PVR & 1.004 & $0.999-1.009$ & 0.154 & & & & & & \\
\hline PV & 1.021 & $1.003-1.039$ & 0.021 & 1.016 & $0.997-1.035$ & 0.102 & - & - & - \\
\hline PSA & 1.215 & $1.048-1.410$ & 0.010 & - & - & - & 1.185 & $1.020-1.378$ & 0.027 \\
\hline
\end{tabular}

Abbreviations: $\mathrm{Cl}$, confidence interval; IPSS, international prostate symptom scores; BPI, BPH problem index; BPH, benign prostatic hyperplasia; QoL, quality of life; PVR, postvoid residual urine volume; PV, prostate volume; PSA, prostate-specific antigen; $Q_{\max }$, maximum flow rate. 
A

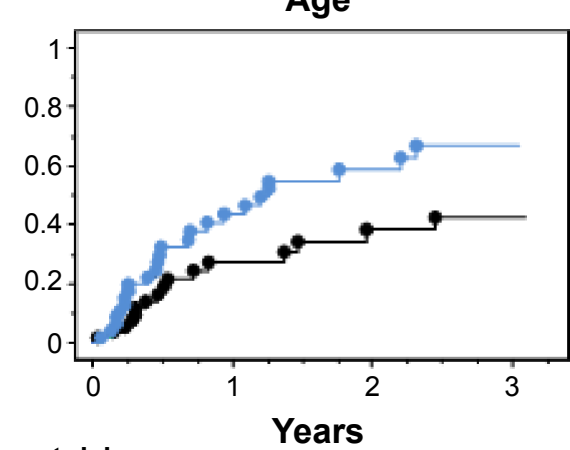

Number at risk

$\begin{array}{lllllll}\geq 70 \text { years } & 543930 & 21 & 14 & 11 & 9 & 7 \\ <70 \text { years } & 634936 & 25 & 20 & 17 & 16 & 13\end{array}$
B

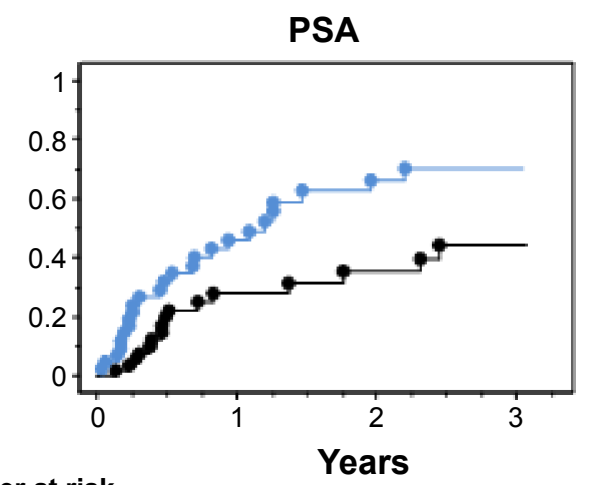

Number at risk

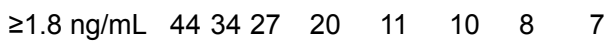

$<1.8 \mathrm{ng} / \mathrm{mL} \quad 695137 \quad 24 \quad 21 \quad 16 \quad 15 \quad 11$

C

PV

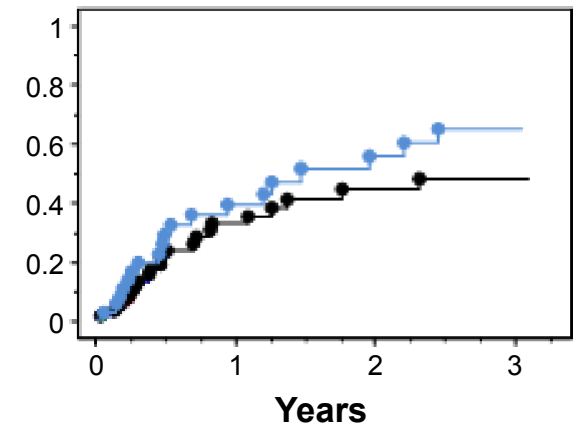

Number at risk

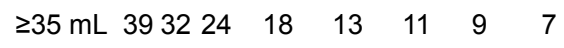

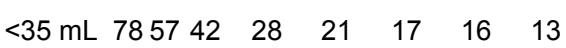

Figure 2 Rates of treatment failure by age, PV, and the values of serum PSA at baseline with naftopidil for 3-year follow-up determined by the Kaplan-Meier curve. Notes: (A) Age: blue and black lines indicate patients aged $\geq 70$ years $(n=54)$ and those aged $<70$ years $(n=63)$, respectively, $P=0.028$. (B) PSA: blue and black lines indicate patients with PSA $\geq 1.8 \mathrm{ng} / \mathrm{mL}(\mathrm{n}=44)$ and those with $\mathrm{PSA}<1.8 \mathrm{ng} / \mathrm{mL}(\mathrm{n}=69)$, respectively, $P=0.008$. (C) $P V$ : blue and black lines indicate patients with $P V \geq 35 \mathrm{~mL}$ ( $\mathrm{n}=39$ ) and those with $\mathrm{PV}<35 \mathrm{~mL}(\mathrm{n}=78)$, respectively, $\mathrm{P}=0.226$.

Abbreviations: PV, prostate volume; PSA, prostate-specific antigen.

\section{Discussion}

Naftopidil, with a threefold higher affinity for $\alpha 1 \mathrm{D}$ - than for the $\alpha 1 \mathrm{~A}$-adrenergic receptor subtype, is an $\alpha 1$-blocker that has been approved for clinical use for BPH/LUTS in Japan since $1999 .{ }^{3}$ It is also currently available in Korea,

Table 3 Results of outcome survey at 3 years in 50 patients who quit medication during the follow-up period

\begin{tabular}{ll}
\hline Outcome & $\begin{array}{l}\text { Number of } \\
\text { patients (\%) }\end{array}$ \\
\hline $\begin{array}{l}\text { No events } \\
\text { Retreatment }\end{array}$ & $37(74)$ \\
$\quad$ Retreatment with $\alpha$ I-blockers & $10(20)$ \\
$\quad$ Surgery after retreatment with $\alpha$ I-blockers & $3(6)$ \\
AUR & $0(0)$ \\
Total & 50 \\
\hline
\end{tabular}

Abbreviation: AUR, acute urinary retention.
Table 4 Comparison of baseline parameters between 33 patients who quit naftopidil due to improvement in LUTS and the remaining 84 patients

\begin{tabular}{|c|c|c|c|}
\hline \multirow[t]{2}{*}{ Parameters } & \multirow{2}{*}{$\begin{array}{l}\text { Improvement } \\
\text { in LUTS } \\
\mathrm{n}=33\end{array}$} & \multirow{2}{*}{$\begin{array}{l}\text { Remaining } \\
\text { patients } \\
\mathbf{n}=\mathbf{8 4}\end{array}$} & \multirow[t]{2}{*}{$P$-value } \\
\hline & & & \\
\hline Age & $67.0 \pm 6.3$ & $68.8 \pm 6.7$ & 0.194 \\
\hline IPSS & $16.0 \pm 6.4$ & $18.2 \pm 5.5$ & 0.066 \\
\hline QoL index & $4.6 \pm 0.9$ & $4.9 \pm 0.9$ & 0.206 \\
\hline BPI & $12.2 \pm 6.0$ & $13.2 \pm 5.6$ & 0.408 \\
\hline$Q_{\max }$ & $13.6 \pm 5.8$ & $10.5 \pm 4.7$ & 0.004 \\
\hline PVR & $54.7 \pm 48.2$ & $68.7 \pm 69.1$ & 0.291 \\
\hline PV & $29.0 \pm 8.5$ & $35.0 \pm 14.6$ & 0.027 \\
\hline PSA & $1.3 \pm 1.6$ & $1.9 \pm 1.5$ & 0.051 \\
\hline \multicolumn{4}{|c|}{$\begin{array}{l}\text { Notes: Differences in parameters between patients with improvement in LUTS and } \\
\text { the remaining patients were determined by unpaired } t \text {-test. Data are presented as } \\
\text { mean } \pm \text { standard deviation. }\end{array}$} \\
\hline \multicolumn{4}{|c|}{$\begin{array}{l}\text { Abbreviations: IPSS, international prostate symptom scores; QoL, quality of life; } \\
\text { BPI, BPH problem index; BPH, benign prostatic hyperplasia; PVR, postvoid residual } \\
\text { urine volume; PV, prostate volume; PSA, prostate-specific antigen; LUTS, lower } \\
\text { urinary tract symptoms; } Q \text {, maximum flow rate. }\end{array}$} \\
\hline
\end{tabular}


Table 5 Changes in IPSS in 33 patients whose reason for discontinuation was improvement in LUTS

\begin{tabular}{|c|c|c|c|}
\hline Parameters & $\begin{array}{l}\text { No events } \\
(n=25)\end{array}$ & $\begin{array}{l}\text { Retreatment } \\
(n=8)\end{array}$ & $P$-value \\
\hline \multicolumn{4}{|l|}{ IPSS } \\
\hline Baseline & $15.9 \pm 6.9$ & $16.4 \pm 4.7$ & 0.864 \\
\hline Last visit & $5.4 \pm 3.2 * *$ & $7.2 \pm 4.8^{* * *}$ & 0.370 \\
\hline After discontinuation & $5.7 \pm 4.3^{*}$ & $5.8 \pm 8.5 * * * *$ & 0.967 \\
\hline \multicolumn{4}{|l|}{ QoL index } \\
\hline Baseline & $4.5 \pm 0.8$ & $5.0 \pm 1.2$ & 0.209 \\
\hline Last visit & $1.4 \pm 0.8^{*}$ & $2.0 \pm 1.1 * * *$ & 0.191 \\
\hline After discontinuation & $2.6 \pm 1.3 * *, * * * * *$ & $2.3 \pm 1.4^{* * * * *}$ & 0.643 \\
\hline \multicolumn{4}{|l|}{ BPI } \\
\hline Baseline & $12.6 \pm 6.0$ & $10.7 \pm 6.0$ & 0.462 \\
\hline Last visit & $3.1 \pm 3.3^{* *}$ & $5.0 \pm 4.2$ & 0.356 \\
\hline After discontinuation & $4.8 \pm 4.2 * * *$ & $6.2 \pm 5.8$ & 0.551 \\
\hline
\end{tabular}

Notes: $* P<0.0001, * * P<0.001, * * * P<0.01$, $* * * * P<0.05$ vs baseline (paired $t$-test). No differences were observed in the IPSS, QoL index, and BPI between the last visit and after discontinuation in both no events and retreatment groups, except in the QoL index in the no events group (***** $P=0.01$ I vs last visit [paired $t$-test]). Times between baseline and the last visit were $11.0 \pm 11.5$ months and $5.1 \pm 2.1$ months in the no events and retreatment groups, respectively $(P=0.166)$. Times between the last visit and after discontinuation were $16.7 \pm 11.5$ months and $13.1 \pm 4.0$ months in the no events and retreatment groups, respectively $(P=0.435)$. Differences in parameters between no events and retreatment were determined by unpaired $t$-test. Data are presented as mean \pm standard deviation.

Abbreviations: IPSS, international prostate symptom scores; LUTS, lower urinary tract symptoms; QoL, quality of life; BPI, BPH problem index; $\mathrm{BPH}$, benign prostatic hyperplasia.

the People's Republic of China, and India. The short-term efficacy and safety of naftopidil are well known and summarized in the several recent review articles. ${ }^{4-8}$ On the other hand, information on the long-term efficacy and safety of naftopidil monotherapy is extremely limited. ${ }^{9,10}$ To our knowledge, the present study is the first report to prospectively evaluate the long-term outcome of naftopidil at 3 years.

In this study, the total IPSS, QoL index, BPI, and $Q_{\max }$ were significantly improved during 3 years by naftopidil (Table 1). Not only voiding symptoms but also storage symptoms showed significant improvements. Only $5 \%$ of the patients quitted the medication because of adverse events, mainly due to postural hypotension. Thus, the long-term efficacy and safety of naftopidil were clearly demonstrated to be as good as those of other $\alpha 1$-blockers such as tamsulosin. ${ }^{1}$ However, only 25 patients $(21.4 \%)$ continued the same medication for 3 years. The results were comparable to our previous retrospective study indicating that only $19 \%$ of patients continued taking naftopidil at 4 years. ${ }^{9}$ Similarly, our previous prospective study using tamsulosin demonstrated that two-thirds of the men to whom tamsulosin was given terminated the medication by 5 years. ${ }^{1}$ Thus, we could confirm that all men who receive monotherapy with $\alpha 1$-blockers such as tamsulosin and naftopidil do not necessarily continue the same medication for a long time.
There were various reasons for discontinuation (Figure 1). For-cause termination, including treatment failure and loss to follow-up, was almost the same as in the tamsulosin study. ${ }^{1}$ It has been reported that there is no significant difference in the proportion of self-discontinuation between naftopidil and tamsulosin. ${ }^{10}$ In addition, $29 \%$ of men who received naftopidil quitted the medication due to symptomatic improvement. This is also comparable to the $19 \%$ observed in the tamsulosin study. ${ }^{1}$ Thus, one-fifth to one-third of the patients using $\alpha 1$-blockers showed improvement in LUTS after short-term medication. Even after termination of the drug, LUTS evaluated by the IPSS, QoL index, and BPI were stable for $\sim 1$ year (Table 5), similar to the results of the tamsulosin study. ${ }^{1}$ Considering factors predicting symptomatic improvement in the tamsulosin study, the 21 patients who terminated the drug because of symptomatic improvement were younger and had lower PSA at baseline than the remaining 91 patients. ${ }^{1}$ The PV of the former was smaller than that of the latter although there was no significant difference. In the present study, baseline $Q_{\max }$ and PV were significantly different between the two groups (Table 4). Although there were no significant differences, the IPSS and PSA showed marginal significance. Thus, patients who had less severe subjective and objective symptoms at baseline were likely to achieve symptomatic improvement during follow-up in short-term $\alpha 1$-blocker monotherapy.

One of the main purposes of the present study was to determine the status of retreatment after discontinuation of naftopidil for various reasons, including symptomatic improvement, as it has not been evaluated in detail previously. Only $26 \%$ of the 50 men who quitted the medication needed retreatment with an $\alpha 1$-blocker and/or surgery (Table 3). In the 33 patients whose reason for discontinuation was symptomatic improvement, there were no differences in baseline age, PV, and PSA between the eight patients with retreatment and the 25 patients with no events. Yokoyama et $\mathrm{al}^{11}$ reported changes in subjective and objective symptoms after discontinuation of $\alpha 1$-blockers in 60 patients who agreed to stop the drugs once their symptoms improved. During follow-up, 18 (30\%) patients required retreatment within 12 months after discontinuation, whereas 42 patients (70\%) maintained good urinary condition without retreatment for 12 months. Although they reported that the PV was smaller and the serum PSA value was lower just before discontinuation in patients without retreatment than in those with it, our study failed to identify factors at baseline and the last visit predicting subsequent retreatment. However, the majority of men who quit medication due to symptomatic improvement 
may have durable improvement in LUTS even after termination of $\alpha 1$-blockers.

In the present study, treatment failure was observed in $35 \%$ of the patients during 3 years, whereas it occurred in $18.8 \%$ during 5 years in the tamsulosin study. ${ }^{1}$ However, the definition of treatment failure was different between the studies. In the tamsulosin study, it was defined as disease progression (PVR $\geq 200 \mathrm{~mL}$, AUR, febrile urinary tract infection, or hydronephrosis due to bladder outlet obstruction with or without azotemia), conversion to another $\alpha 1$-blocker, or conversion to surgery. Since the baseline PVR amount was not one of the inclusion criteria for the present study, some patients had PVR of $\geq 200 \mathrm{~mL}$ even at baseline. In addition, since the $5 \alpha$-RI dutasteride was approved in Japan in 2009, some patients whose $\mathrm{PV}$ was $\geq 30 \mathrm{~mL}$ had a chance to receive add-on of dutasteride during follow-up if naftopidil monotherapy was ineffective. Furthermore, the Medical Therapy of Prostatic Symptoms (MTOPS) ${ }^{12}$ and the Combination of Avodart ${ }^{\mathbb{B}}$ and Tamsulosin (CombAT) ${ }^{13}$ studies used symptom deterioration of the IPSS $\geq 4$ points as a parameter on two consequent visits to indicate clinical progression, although we did not confirm it by readministration of the IPSS within 4 weeks. Thus, it is impossible to directly compare the results derived from these two prospective studies. However, quite a few patients had treatment failure of $\alpha 1$-blocker monotherapy during the long-term follow-up, as indicated in larger studies. ${ }^{12,13}$

Finally, we analyzed risk factors that could predict treatment failure. In the tamsulosin study, ${ }^{1}$ although several dynamic variables after treatment such as the changes in the IPSS and QoL index at 3 months were evaluated, only static variables at baseline were evaluated in the present study because a substantial number of the patients dropped out during the first 3 months. The PV at baseline was not a significant factor for treatment failure, although PSA, which is a surrogate marker for PV, was the sole factor in the multivariate analysis. Previously, we reported that baseline PV was the most frequently observed and/or the strongest static variable to predict treatment failure of $\alpha 1$-blockers according to a prospective 5 -year tamsulosin study ${ }^{1}$ and a retrospective 4-year naftopidil study. ${ }^{9}$ This difference in results may be due to the differences in the follow-up period. In the MTOPS study, the curves of the cumulative incidences of AUR and invasive therapy for BPH in a doxazosin arm and a finasteride arm started to separate from 2.5 years to 3 years after baseline. ${ }^{12}$ Thus, 3 years may not be long enough to evaluate the long-term outcome of $\alpha 1$-blocker monotherapy, which is probably affected by baseline PV.
Conclusion

This is the first study to prospectively evaluate the clinical courses of patients who received naftopidil for BPH/LUTS, including those who dropped out during follow-up. Data were also collected from the patients who quit medication at the time point scheduled as the final examination at 3 years. Although the small number of patients enrolled and two initial dosage of naftopidil used are major limitations, the study provides information on the long-term clinical outcome of naftopidil monotherapy for patients with BPH/LUTS as well as risk factors for treatment failure.

\section{Acknowledgments}

The authors thank members of the Hokkaido Urological Disorders Conference for their assistance: Doctor Satoshi Takahashi and Doctor Ryuichi Kato of Sapporo Medical University, Doctor Koichi Takeda and Doctor Nobukazu Suzuki of Teine Urological Clinic, Doctor Toshikazu Nitta of Sanjukai Hospital, Doctor Masahiro Nishimura of Motomachi Urology, Doctor Naoto Mikuma of Bethel Urology Clinic, Doctor Masanori Matsukawa of Takikawa Municipal Hospital, Doctor Yoshinori Tanaka of Hokkaido Prefectural Esashi Hospital, and Doctor Masamichi Sasai of Yawaragi Hinyokika. A part of this paper was presented at the AUA Annual Meeting 2014 as a poster presentation. The poster's abstract was published in J Urol. 191(4):e671, supplement (hyperlinks: http:// www.jurology.com/article/S0022-5347(14)02107-7/abstract and https://www.auanet.org/university/abstract detail.

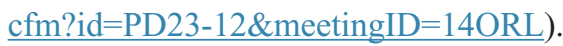

\section{Author contributions}

All authors contributed toward data analysis, drafting and critically revising the paper and agree to be accountable for all aspects of the work.

\section{Disclosure}

Naoya Masumori has received advisor fee and lecture fee from Astellas Pharma. Naoya Masumori has also received lecture fees from Nippon Shinyaku, Asahi Kasei, and GSK. Taiji Tsukamoto, Akihiko Shibuya, Noriomi Miyao, Yasuharu Kunishima, and Akihiko Iwasawa declare that they have no conflicts of interest in this work.

\section{References}

1. Masumori N, Tsukamoto T, Horita H, et al. $\alpha 1$-blocker tamsulosin as initial treatment for patients with benign prostatic hyperplasia: 5-year outcome analysis of a prospective multicenter study. Int J Urol. 2013; 20(4):421-428.

2. Collins GN, Raab GM, Hehir M, King B, Garraway WM. Reproducibility and observer variability of transrectal ultrasound measurements of prostate volume. Ultrasound Med Biol. 1995;21(9):1101-1105. 
3. Takei R-I, Ikegaki I, Shibata K, Tsujimoto G, Asano T. Naftopidil, a novel $\alpha 1$-adrenoceptor antagonist, displays selective inhibition of canine prostatic pressure and high affinity binding to cloned human

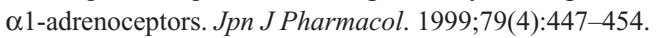

4. Garimella PS, Fink HA, MacDonald R, Wilt TJ. Naftopidil for the treatment of lower urinary tract symptoms compatible with benign prostatic hyperplasia (review). Cochrane Database Syst Rev. 2009;(4): CD007360.

5. Masumori N. Naftopidil for the treatment of urinary symptoms in patients with benign prostatic hyperplasia. Ther Clin Risk Manag. 2011; 7:227-238.

6. Hara N, Mizusawa T, Obara K, Takahashi K. The role of naftopidil in the management of benign prostatic hyperplasia. Ther Adv Urol. 2013;5(2): 111-119.

7. Fukuta F, Masumori N. A review of naftopidil for treatment of lower urinary tract symptoms suggestive of benign prostatic hyperplasia. Curr Bladder Dysfunct Rep. 2015;10(2):160-169.

8. Castiglione F, Benigni F, Briganti A, et al. Naftopidil for the treatment of benign prostate hyperplasia: a systematic review. Curr Med Res Opin. 2014;30(4):719-732.

9. Masumori N, Hashimoto J, Itoh N, Tsukamoto T, Group TS. Shortterm efficacy and long-term compliance/treatment failure of the alpha1 blocker naftopidil for patients with lower urinary tract symptoms suggestive of benign prostatic hyperplasia. Scand J Urol Nephrol. 2007; 41(5):422-429.
10. Kawachi Y, Sakurai T, Sugimura S, et al. Long-term treatment and prognostic factors of alpha 1-blockers for lower urinary tract symptoms associated with benign prostatic hyperplasia: a pilot study comparing naftopidil and tamsulosin hydrochloride. Scand J Urol Nephrol. 2010; 44(1):38-45.

11. Yokoyama T, Watanabe T, Saika T, et al. Natural course of lower urinary tract symptoms following discontinuation of alpha-1-adrenergic blockers in patients with benign prostatic hyperplasia. Int J Urol. 2007;14(7): 598-601.

12. McConnell JD, Roehrborn CG, Bautista OM, et al; Medical Therapy of Prostatic Symptoms (MTOPS) Research Group. The long-term effect of doxazosin, finasteride, and combination therapy on the clinical progression of benign prostatic hyperplasia. N Engl J Med. 2003;349(25): 2387-2398.

13. Roehrborn CG, Siami P, Barkin J, et al; CombAT Study Group. The effects of combination therapy with dutasteride and tamsulosin on clinical outcomes in men with symptomatic benign prostatic hyperplasia: 4-year results from the CombAT study. Eur Urol. 2010;57(1): $123-131$.

\section{Publish your work in this journal}

Patient Preference and Adherence is an international, peer-reviewed, open access journal that focuses on the growing importance of patient preference and adherence throughout the therapeutic continuum. Patient satisfaction, acceptability, quality of life, compliance, persistence and their role in developing new therapeutic modalities and compounds to optimize clinical outcomes for existing disease states are major areas of interest for the journal. This journal has been accepted for indexing on PubMed Central. The manuscript management system is completely online and includes a very quick and fair peer-review system, which is all easy to use. Visit http://www dovepress.com/testimonials.php to read real quotes from published authors. 